\title{
Mathematical Modelling of Process of Shaping of Sand-Pitch Mixes
}

\author{
Vitaliy Yu. Kulikov*a, Evgeniy N. Eremin', \\ Tatyana V. Kovalyova ${ }^{b}$ and Elena P. Scherbakova ${ }^{a}$ \\ ${ }^{a}$ Karaganda State Technical University \\ 56 Boulvard Mira, Karaganda, 100027, Republic of Kazakhstan \\ ${ }^{b}$ Omsk State Technical University \\ 11 Mira, Omsk, 644050, Russia
}

Received 11.01.2018, received in revised form 10.08.2019, accepted 17.09.2019

This article considers mathematical dependences of properties of a casting mold on various parameters of her shaping. The competitiveness in production of castings depends on durability, reliability of the made details, ability to meet requirements and expectations of the consumer of production. Technological process of receiving castings most widespread now in the sandyargillaceous forms (SAF) not completely meets the modern requirements as it is characterized by different types of marriage: gas porosity, scorch, shrinkable sinks, blockages, hot and cold cracks, and others. The bigger quality of castings gives casting in the sand-pitch forms (SPF) in which the high gas permeability and durability are combined, they don't resist shrinkage, don't absorb moisture the stiffening alloy and aren't inclined to an fallibility. However, one of shortcomings of this way of casting is rather high cost binding. One of the directions of decrease in an expense binding is use along with heating of mix for shaping of a cover and static loading. Other direction of decrease in a consumption of mix in general, and binding in particular, is determination of dependence of properties of a shell form on technological parameters that will promote management of properties of a form, decrease in marriage, etc. Formulas of the intense deformed state, density, amounts of heat of the sand-pitch form made at the same time in the conditions of heating and static loading are presented.

Keywords: model, form, sand, resin, technology, casting, mixture.

Citation: Kulikov V.Yu., Eremin E.N., Kovalyova T.V., Scherbakova E.P. Mathematical modelling of process of shaping of sand-pitch mixes, J. Sib. Fed. Univ. Eng. technol., 2019, 12(7), 810-817. DOI: 10.17516/1999-494X-0181.

(C) Siberian Federal University. All rights reserved

This work is licensed under a Creative Commons Attribution-NonCommercial 4.0 International License (CC BY-NC 4.0).

* Corresponding author E-mail address: mlpikm@mail.ru 


\title{
Математическое моделирование процесса формообразования песчано-смоляных смесей
}

\author{
В.Ю. Куликов ${ }^{a}$, Е.Н. Еремин ${ }^{0}$, \\ Т.В. Ковалева ${ }^{\sigma}$, Е.П. Щербакова ${ }^{a}$ \\ ${ }^{a}$ Карагандинский государственный технический университет \\ Республика Казахстан, 100027, Караганда, Бульвар Мира, 56 \\ ${ }^{6}$ Омский государственный технический университет \\ Россия, 644050, Омск, пр. Мира, 11
}

\begin{abstract}
Эта статья рассматривает математические зависимости свойств литейной формы от различных параметров ее формообразования. Конкурентоспособность в производстве отливок зависит от долговечности, надежности производимых деталей, способности удовлетворить требования и ожидания потребителя продукиии. Наиболее распространенный в настоящее время технологический процесс получения отливок в песчано-глинистые формы (ПГФ) не полностью отвечает современным требованиям, поскольку характеризуется различными видами брака: газовая пористость, пригар, усадочные раковины, засоры, горячие и холодные трещчины и др. Большее качество отливок дает литье в песчаносмоляные формы (ПСФ), в которых сочетаются высокая газопроницаемость и прочность, они не сопротивляются усадке, не впитывают влагу застывающим сплавом и не склоннь к осыпаемости. Однако одним из недостатков этого способа литья является относительно высокая стоимость связуюшего. Одним из направлений снижения расхода связующего служит использование одновременно с нагревом смеси для формообразования оболочки и статической нагрузки. Другое направление снижения расхода смеси в иелом и связующего в частности - определение зависимости свойств оболочковой формы от технологических параметров, что будет способствовать управлению свойствами формьл, снижению брака u m.n. Представлень формуль напряженно-деформированного состояния, плотности, количества тепла песчано-смоляной формы, изготавливаемой одновременно в условиях нагрева и статического нагружения.
\end{abstract}

Ключевые слова: модель, форма, песок, смола, технология, отливка, смесь.

От долговечности и эффективности работы промышленного оборудования напрямую зависят сроки и качество выполняемых работ, а также затраты на его эксплуатацию. Значит, обеспечение заготовительной и ремонтной базы промышленности качественными деталями является первостепенной задачей.

Конкурентоспособность в производстве отливок зависит от долговечности, надежности производимых деталей, способности удовлетворить требования и ожидания потребителя продукции.

При этом при изготовлении деталей важно снизить затраты на их производство, обеспечив долговечность и надежность.

Таким образом, использование новых технологий для производства долговечных отливок - задача стратегическая.

Наиболее распространенный в настоящее время технологический процесс получения отливок в песчано-глинистые формы (ПГФ) не полностью отвечает современным требованиям, поскольку характеризуется различными видами брака: газовая пористость, пригар, усадочные раковины, засоры, горячие и холодные трещины и др. Кроме того, литье в ПГФ не всегда обе- 
спечивает получение отливок с требуемой структурой и, соответственно, с необходимым уровнем технологических свойств.

Большее качество отливок дает литье в песчано-смоляные формы (ПСФ), в которых сочетаются высокая газопроницаемость и прочность, они не сопротивляются усадке, не впитывают влагу застывающим сплавом и не склонны к осыпаемости. Также они с малыми энергозатратами разрушаются после формирования отливки. Это обеспечивает получение отливок, обладающих высокой чистотой поверхности и размерной точностью, а также большую экономию формовочных материалов (по сравнению с песчано-глинистыми формами более 50 \%). В свою очередь, уменьшение оборота формовочных материалов значительно снижает расходы по внутризаводской и внешней транспортировке. При использовании литья в оболочковые формы увеличивается выход годного [1-3].

Однако один из недостатков этого способа литья - относительно высокая стоимость связующего. Одним из направлений снижения расхода связующего является использование одновременно с нагревом смеси для формообразования оболочки и статической нагрузки $[4,5]$. Другим направлением снижения расхода смеси в целом и связующего в частности служит пределение зависимости свойств оболочковой формы от технологических параметров, что будет способствать управлению свойствами формы, снижению брака и т.п.

Цель настоящего исследования - разработка матматических зависимостей для оперативного управления свойства оболочковой формы.

Ранее [6] было определено напряжение релаксации $\sigma_{p}$ песчано-смоляной смеси:

$$
\varepsilon=\left(-\frac{2 \cdot \tau_{1}}{E_{0}} \cdot e^{-\frac{t}{\tau_{1}}}\right) \cdot \sigma+\left(\frac{2 \cdot \tau_{1}}{E_{0}}\right) \cdot \sigma=\sigma \cdot\left[\frac{2 \cdot \tau_{1}}{E_{0}} \cdot\left(1-e^{-\frac{t}{\tau_{1}}}\right)\right] .
$$

Формула (1) является уравнением ползучести ПСС в условиях формообразования со статической нагрузкой.

$$
\sigma_{p}=\frac{E_{0} \cdot E_{1} \cdot \varepsilon_{p}}{2 \cdot\left(E_{0}+E_{1}\right)}
$$

где $E_{0}, E_{1}$ - соответственно модуль упругости, в момент времени $t=0$ и $t=t_{l} ; \varepsilon_{p}$ - деформация релаксации.

Сравнение теоретических и практических результатов приведено на рис. 1.

На графике показана экспериментальная линия регрессии (кривая 1).

Расчетная линия регрессии (кривая 2) построена по уравнению $\mathrm{y}=-16,667 \mathrm{x}^{2}+4,6333 \mathrm{x}+$ $+0,008$ с коэффициентом достоверности $\mathrm{R}^{2}=0,9799$.

Таким образом, нелинейность деформации ПСС обусловлена различным характером зависимости упругой, вязкой и пластической деформации от напряжения. Получено уравнение ядра ползучести ПСС.

Также была определена формула количества теплоты Q (2), расходуемой на нагрев оболочки:

$$
Q=\delta_{1} \cdot\left[\left(T_{\kappa}-T_{0}\right) / \sum_{n=0}^{n=k} \frac{4 \cdot z_{n}}{\pi \cdot d_{n}^{2} \cdot \lambda_{n}}\right]+\delta_{2} \cdot\left[\left(T_{\kappa}-T_{0}\right) /\left(\sum_{n=0}^{n=k} \frac{1}{\alpha_{n-1}}+\frac{d_{n}}{\lambda_{n}}+\frac{1}{\alpha_{n}} / \frac{3 \cdot \pi \cdot d_{n}^{2}}{4}\right)\right],
$$


где $\delta_{1}, \delta_{2}-$ коэффициенты распределения, зависящие от преобладания конвекции или теплопроводности; $d$ - диаметр в точке соприкосновения частиц твердой фазы; $z_{1}-$ диаметр (высота) одной песчинки; $T_{\kappa}$ и $T_{0}$ - температура подмодельной плиты конечная и начальная; $\lambda$ - теплопроводность дисперсной среды; $a_{2}$ - коэффициент температуропроводности.

На коэффициенты распределения влияет степень уплотнения, форма и размер частиц твердой фазы и другие факторы. В реальных условиях можно принимать за сферическую форму песчинок, учитывать пористость и процентное соотношение смолы и кварцевого песка. С уменьшением пористости смеси уменьшается значение конвективного теплообмена и коэффициент $\delta_{2}$ уменьшается.

Очевидно, что повышение истинной плотности в процессе термического воздействия приведет к увеличению скорости прогрева формы, т. е. к сокращению тепловых потерь. Приложение статической нагрузки в начальный момент нагрева дисперсной смеси приводит к повышению ее плотности [7].

Следует учитывать и то, что по мере плавления смолы (а также увеличения статической нагрузки на формирующуюся дисперсную смесь) смола заполняет поры и твердеет, схватывая песчинки. Здесь уже будет происходить передача тепла теплопроводностью. Для такого состояния значение $\lambda$ будет также зависеть от соотношения связующего и песка.

В формуле (2) $\Delta T$ для конкретных случаев есть величина постоянная, массу смеси можно представить как произведение объема $v$ на плотность $\rho$. Объем дисперсной смеси есть произведение площади $S$ на высоту $z$. Площадь определяется геометрическими размерами модельной нагреваемой плиты, а высота есть толщина оболочковой формы (величина прогрева и твердения формы). Толщину стенки формы $z$ можно вычислить по формуле

$$
z=k \cdot \sqrt{\tau},
$$

где $k$ - коэффициент пропорциональности; $\tau$ - время теплового воздействия на дисперсную песчано-смоляную среду [8].

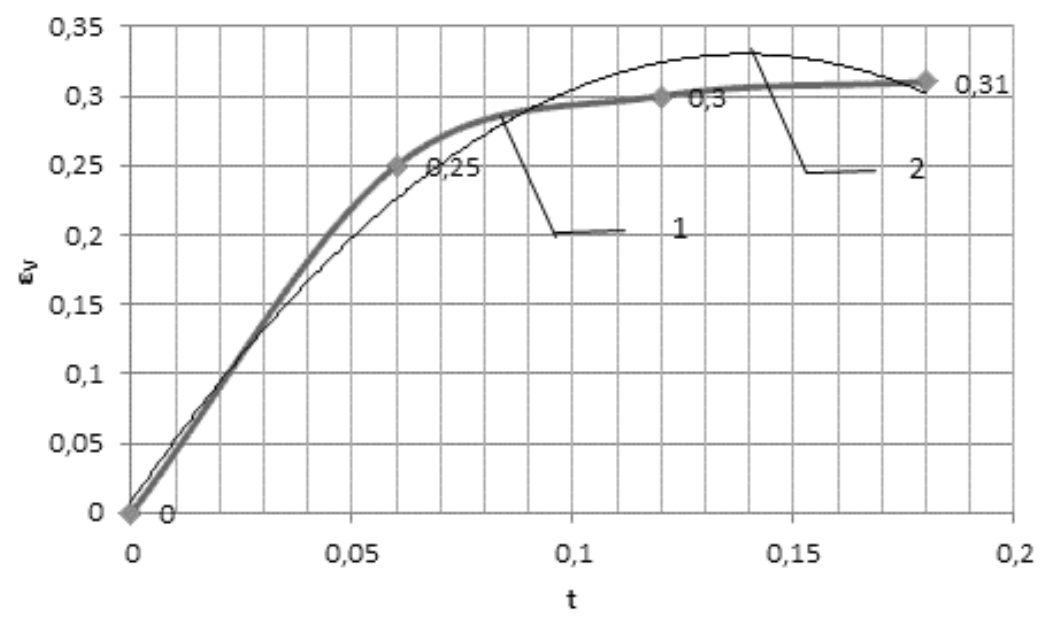

Рис. 1. Сравнение теоретических и экспериментальных данных деформации ПСС во времени

Fig. 1. Comparison of theoretical and experimental data of SRM deformation in time 
Таким образом, можно получит зависимость тепла, идущего на нагрев песчано-смоляной смеси, от времени теплового воздействия на эту смесь:

$$
Q=c \cdot \Delta T \cdot \rho \cdot S \cdot k \cdot \sqrt{\tau} .
$$

В правой части формулы все значения кроме времени есть постоянные (можно изначально задаваться различными температурами нагрева смеси и площадью, подвергаемой термическому воздействию).

Полученную зависимость можно использовать для определения времени нагрева смеси, необходимого для образования оболочковой формы.

На рис. 2 представлена зависимость количества теплоты от времени нагрева (экспериментальные данные и данные расчета по полученной формуле, эксперименты проводили в цехе коркового литья ТОО «КМЗ имени Пархоменко», г. Караганда, Казахстан) при температуре нагрева модельной плиты $250{ }^{\circ} \mathrm{C}$.

Очевидно, что выделение теплоты происходит по закону, близкому к параболическому. Для мелких и средних отливок оптимальная толщина песчано-смоляной оболочки составляет 8-10 мм, для обеспечения такой толщины необходим нагрев смеси в течение 25-30 с. Уменьшение толщины оболочки приведет к разрушению формы при заливке, а увеличение толщины - к перерасходу смеси и ухудшению ее газопроницаемости. Дальнейшее время нагрева нецелесообразно, так как интенсивность выделения теплоты для прогрева формы уменьшается, к тому же происходит выгорание смолы, что разупрочняет форму. Расхождение между экспериментальными и расчетными данными составляет 5-7 \%.

Таким образом, получена зависимость количества теплоты от времени теплового воздействия. Теоретические и экспериментальные исследования показали, что для получения качественной оболочки необходимо нагревать песчано-смоляную смесь в течение 25-30 с при температуре $250{ }^{\circ} \mathrm{C}$. В случае увеличения продолжительности или температуры нагрева происходит разупрочнение формы за счет выгорания связующего (смолы).

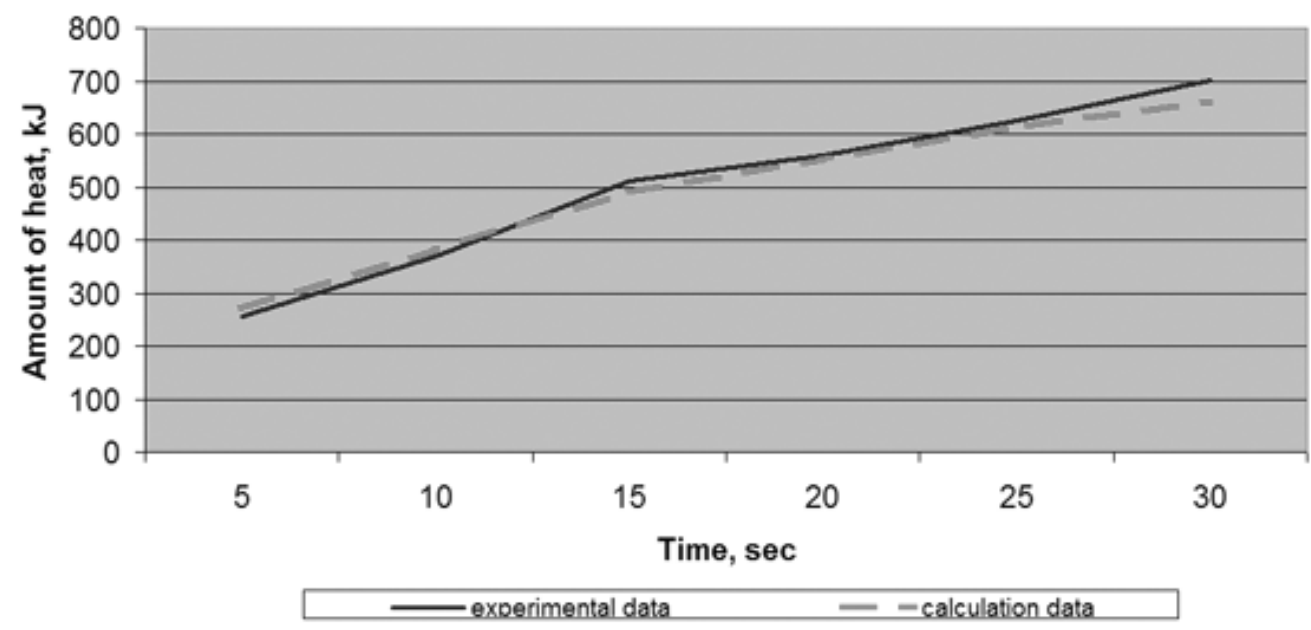

Рис. 2. Зависимость количества теплоты от времени нагрева смеси

Fig. 2. The heat quantity depends on the heating time of the mixture 
Пористость песчано-смоляной смеси можно рассчитать по следующей зависимости:

$$
m=1-\frac{d^{2} \times S^{2} \times F \times \Delta p \times \tau}{96 \times Q \times h \times \eta},
$$

где $Q$ - объем газа, проходящего через образец поперечного сечения $F$ и высоты $h$ за время $\tau$ при перепаде давлений $\Delta p ; d$ - диметр зерна; $S$ - площадь просвета между частицами смеси; $m$ - пористость смеси; $\eta$ - динамическая вязкость газа.

Плотность можно определить по (6):

$$
\rho=\frac{M \times \beta}{\ln \left[1-\frac{M \times \beta}{\rho_{1} \times\left(-\frac{1}{\alpha} \times \ln \left\{\rho_{n p}-\frac{M}{F \times(H-L)}\right\}\right)^{\mu}}\right]},
$$

где $M$ - масса уплотняемой смеси; $\beta$ - параметр, учитывающий влияние внешнего трения; $F$ площадь прессующего органа; $\rho_{n p}$ - предельная плотность сплошного тела; $H$ - начальная высота заполнения формы; $L$ - расстояние, пройденное прессующей колодкой при прессовании формы; $\rho_{1}-$ плотность смеси при давлении 0,1 МПа; $\alpha$ - коэффициент потери сжимаемости; $\mu$ - коэффициент уплотняемости.

Полученная формула дала хорошую сходимость расчетной и практической величины плотности смеси (рис. 3). На оси абсцисс показано первоначальное значение давления.

По результатам исследований была разработана программа расчетов технологических параметров в зависимости от габаритов опоки, температуры и давления на смесь. Интерфейс программы и результаты вычислений представлены на рис. 4, 5.

На базе проведенных теоретических исследований напряженно-деформированного состояния составлена программа расчета технологических параметров изготовления песчаносмоляной формы.

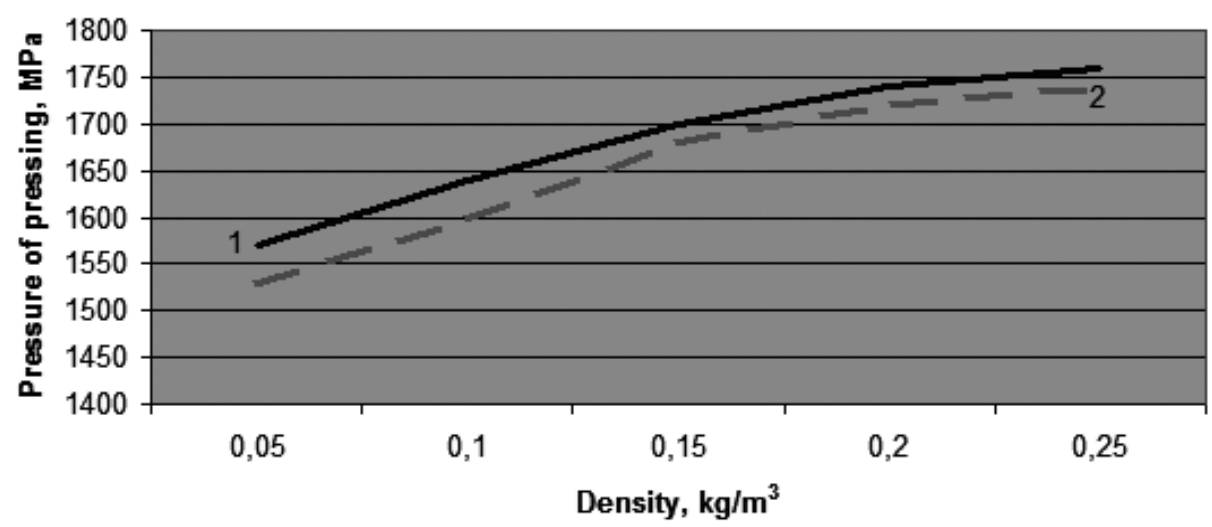

Рис. 3. Расчетная и практическая плотности ПСС в зависимости от давления прессования (1- теория, 2 - эксперимент)

Fig. 3. Influence of pressing pressure on SRM densities (1 - theory, 2 - experiment)

$$
-815-
$$




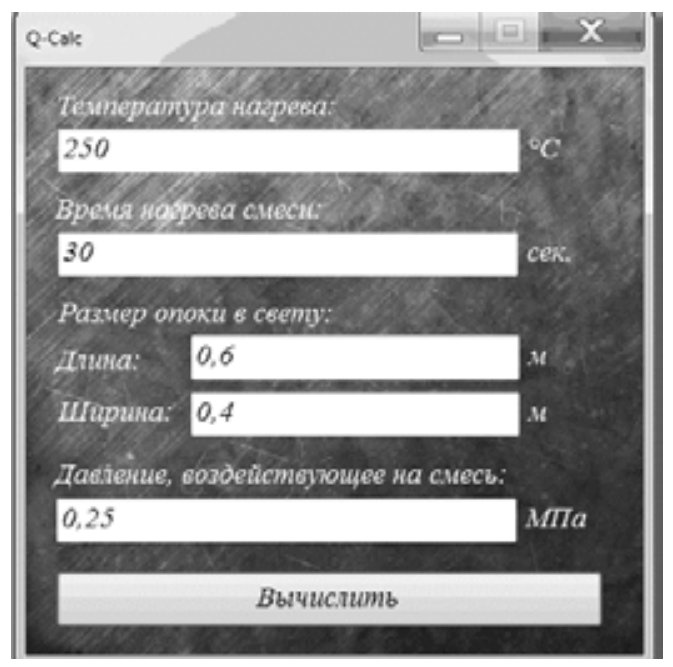

Рис. 4. Пример ввода начальных данных

Fig. 4. Example of initial data introduction

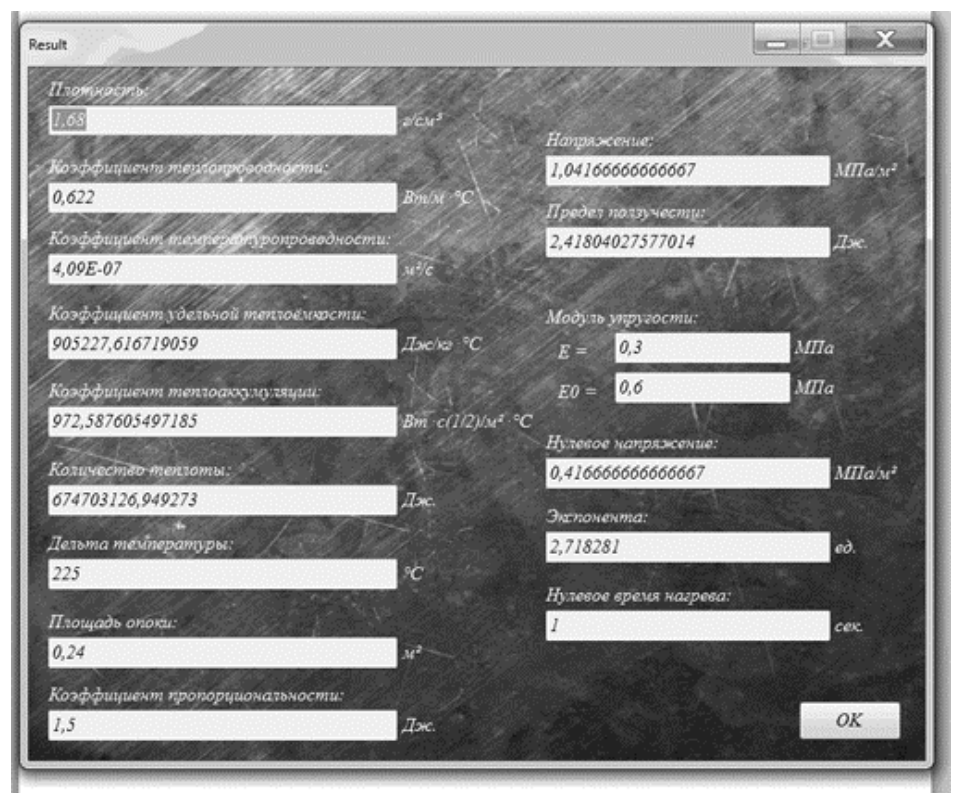

Рис. 5. Результаты вычислений

Fig. 5. Calculation results interface

\section{Список литературы}

[1] Леушина Л.И., Нищенков А.В., Субботин А.Ю. Прогрессивная технология прокаливания оболочковых форм для точного стального литья. Литейщик России, 2011, 2, 40-42 [Leushina L.I., Nishchenkov A.V., Subbotin A.Yu. Progressive technology of calcinating of shell forms for exact steel casting, Founder of Russia. 2011, 2, 40-42 (in Russian)]

[2] Волков Д., Волков А.Д., Ефименко А.В. Литье в оболочковые формы и его универсальность в мелкосерийном и серийном производстве. Литейщик России, 2016, 4, 26-30 [Volkov D., 
Volkov A.D., Efimenko A.V. Casting in shell forms and its universality in small-scale and mass production, Founder of Russia, 2016, 4, 26-30 (in Russian)]

[3] Серебро В.С., Цвиткис Э.Ш., Лысенко Т.В., Давыдова Е.А., Соловьева В.С. Повышение эффективности технологии литья в оболочковые формы. Литейное производство, 1991, 9, 17-19 [Serebro V.S., Tsvitkis E.Sh., Lysenko T.V., Davydova E.A., Solovyova V.S. Increase in efficiency of technology of casting in shell forms, Foundry production, 1991, 9, 17-19 (in Russian)]

[4] Оболенцев Ф.Д., Надземов О.Н., Становский А.Л. Об уплотнении песчано-смоляных оболочковых форм. Литейное производство, 1979, 8, 18-20 [Obolentsev F.D., Nadzemov O.N., Stanovsky A.L. About consolidation of sand-pitch shell forms, Foundry production, 1979, 8, 18-20 (in Russian)]

[5] Зыков А.П. Способы экономии смолы при изготовлении оболочковых форм без ухудшения их несущей способности, Тракторы и сельхозмашины, 1985, 10, 52-54 [Zykov A.P. Ways of economy of pitch at production of shell forms without deterioration in their bearing ability, Tractors and the agricultural machinery, 1985, 10, 52-54 (in Russian)]

[6] Куликов В.Ю., Квон С.С., Исагулов А.З., Ковалева Т.В., Щербакова Е.П. Определение напряжения ползучести и релаксации песчано-смоляных смесей. Фундаментальные исследования, М., 2015, 2 (часть 15), 3272-3274 [Kulikov V.Yu., Kwon S.S., Isagulov A.Z., Kovalyova T.V., Scherbakova E.P. Determination of tension of creep and relaxation of sand-pitch mixes, Basic researches, Moscow, 2015, 2 (part 15), 3272-3274 (in Russian)]

[7] Исагулов А.З., Куликов В.Ю., Щербакова Е.П. Прочность и плотность песчано-смоляных смесей при одновременном статическом и термическом прессовании. Strategiczne pytania swiatowej nauki: труды XI Международной научно-практической конференции. Пржемысль, 7-15 февраля 2015 г, 13-15. [Issagulov A.Z., Kulikov V.Yu., Scherbakova E.P. Durability and density of sand-pitch mixes at simultaneous static and thermal pressing. Strategiczne pytania swiatowej nauki: works XI of the International scientific and practical conference. Przhemysl, on February 7-15, 2015, 13-15 (in Russian)]

[8] Еремин Е.Н., Ковалева Т.В., Куликов В.Ю. Исследование песчано-смоляных смесей при одновременном статическом и термическом воздействии. Омский научный вестник, 2015, 3, 27-29 [Eryomin E.N., Kovalyova T.V., Kulikov of V.Yu. Research of sand-pitch mixes at simultaneous static and thermal influence, the Omsk scientific bulletin, 2015, 3, $27-29$ (in Russian)] 